\title{
Effects of different resistance training frequencies on body composition and muscular performance adaptations in men
}

\author{
Hamid Arazi ${ }^{\text {Corresp., } 1}{ }^{1}$,Abbas Asadi ${ }^{2}$, Paulo Gentil ${ }^{3}$, Rodrigo Ramirez-Campillo ${ }^{4}$, Pooria Jahangiri ${ }^{1}$, Adel Ghorbani ${ }^{1}$, \\ Anthony C Hackney ${ }^{5}$, Hassane Zouhal ${ }^{6}$ \\ ${ }^{1}$ Department of Exercise Physiology, Faculty of Sport Sciences, University of Guilan, Rasht, Guilan, Iran \\ Department of Physical Education and Sport Sciences, Payame Noor University, Rasht, Guilan, Iran \\ 3 Faculdade de Educação Física e Dança, Universidade Federal de Goiás, Goias, Brazil \\ 4 Department of Physical Activity Sciences, Universidad de Los Lagos, Osorno, Chile \\ 5 Department of Exercise \& Sport Science; Department of Nutrition, University of North Carolina, Chapel Hill, North Carolina, United States \\ 6 M2S (Laboratoire Mouvement, Sport, Santé) - EA 1274, Univ Rennes, Rennes, France \\ Corresponding Author: Hamid Arazi \\ Email address: hamid.arazi@guilan.ac.ir
}

Background: The aim of this study was to compare the effects of 8 weeks resistance training (RT) with two sessions versus four sessions per week under volume load-equated conditions on body composition, maximal strength, and explosive actions performance in recreationally trained men.

Methods: Thirty-five healthy young men participated in the study and were randomly divided into a two sessions per-week RT (RT2, $n=12$ ), four sessions per-week RT (RT4, $n=13$ ) or a control group (CG, $n=10)$. All subjects were evaluated for thigh, chest and arm circumference, countermovement jump (CMJ), medicine ball throw (MBT), 1-repetition maximum (1RM) leg press, bench press, arm curl, muscular endurance (i.e., $60 \%$ of 1RM to failure) for leg press, and bench press at pre, mid (week 4) and post an 8week training intervention.

Results: A two-way analysis of variance with repeated measures (3 [group] x 3 [time]) revealed that both training groups increased chest and thigh circumferences, strength and explosive actions performance tests in comparison to CG following 8 weeks of training ( $p=0.01$ to 0.04 ). Group $\times$ time interactions were also noted in 1 RM bench press (effects size $[E S]=1.07$ vs. 0.89 ) and arm curl (ES = 1.15 vs. 0.89$)$, with greater gains for RT4 than RT2 ( $p=0.03)$.

Conclusion: RT improved muscle strength, explosive actions performance and markers of muscle size in recreationally trained men; however, four sessions of resistance training per week produced greater gains in muscular strength for the upper body measures (i.e, 1RM bench press and arm curl) when compared to two sessions per week under volume-equated conditions. 
1 Effects of different resistance training frequencies on body composition and muscular

2 performance adaptations in men

3 Short Title: Weekly Frequency of Resistance Training on Muscular Adaptations

4

5

6 Hamid Arazi ${ }^{*}$, Abbas Asadi², Paulo Gentil ${ }^{3}$, Rodrigo Ramirez-Campillo ${ }^{4}$, Pooria

7 Jahangiri $^{1}$, Adel Ghorbani ${ }^{1}$, Anthony C. Hackney ${ }^{5}$, Hassane Zouhal ${ }^{6}$

$8{ }^{1}$ Department of Exercise Physiology, Faculty of Sport Sciences, University of Guilan, Rasht, Iran.

$9 \quad{ }^{2}$ Department of Physical Education and Sport Sciences, Payame Noor University, Rasht, Iran.

$10{ }^{3}$ Faculdade de Educação Física e Dança, Universidade Federal de Goias, Goias, Brasil.

$11{ }^{4}$ Human Performance Laboratory. Quality of Life and Wellness Research Group. Department of Physical

12 Activity Sciences. Universidad de Los Lagos. Osorno, Chile.

13

14

15

16

17

18

19

20

21

22

23

24

25

26

27

28

${ }^{5}$ Department of Exercise \& Sport Science; Department of Nutrition, University of North Carolina, Chapel Hill, NC, United States.

${ }^{6}$ Univ Rennes, M2S (Laboratoire Mouvement, Sport, Santé) - EA 1274, Rennes, France.

*Corresponding author: Hamid Arazi (Ph.D)

${ }^{1}$ Department of Exercise Physiology, Faculty of Sport Sciences, University of Guilan, P.O. Box: 41635-1438, Rasht, Iran.

E-mail: hamid.arazi@guilan.ac.ir $\quad T e l:+98911-1399207 \quad$ Fax: +98 13-33690675 


\section{Abstract}

Background: The aim of this study was to compare the effects of 8 weeks resistance training (RT) with two sessions versus four sessions per week under volume load-equated conditions on body composition, maximal strength, and explosive actions performance in recreationally trained men.

Methods: Thirty-five healthy young men participated in the study and were randomly divided into a two sessions per-week RT (RT2, n=12), four sessions per-week RT (RT4, n=13) or a control group (CG, $\mathrm{n}=10)$. All subjects were evaluated for thigh, chest and arm circumference, countermovement jump (CMJ), medicine ball throw (MBT), 1-repetition maximum (1RM) leg press, bench press, arm curl, muscular endurance (i.e., $60 \%$ of 1 RM to failure) for leg press, and bench press at pre, mid (week 4) and post an 8-week training intervention.

Results: A two-way analysis of variance with repeated measures (3 [group] x 3 [time]) revealed that both training groups increased chest and thigh circumferences, strength and explosive actions performance tests in comparison to $\mathrm{CG}$ following 8 weeks of training ( $\mathrm{p}=0.01$ to 0.04 ). Group $\times$ time interactions were also noted in 1RM bench press $($ effects size $[E S]=1.07$ vs. 0.89$)$ and arm curl (ES $=1.15$ vs. 0.89$)$, with greater gains for RT4 than RT2 $(p=0.03)$. Conclusion: RT improved muscle strength, explosive actions performance and markers of muscle size in recreationally trained men; however, four sessions of resistance training per week produced greater gains in muscular strength for the upper body measures (i.e, 1RM bench press and arm curl) when compared to two sessions per week under volume-equated conditions.

Keywords: Athletic performance, Body composition, Human physical conditioning, Recovery,

51 Strength training 


\section{INTRODUCTION}

55 Resistance training (RT) is an exercise modality commonly used to improve muscle hypertrophy and strength (ACSM, 2009; Fleck \& Kraemer, 2004). Designing an optimum RT program requires controlling variables such as the number of sets, repetitions, intensity, exercise selection-sequence, and rest intervals (Fleck \& Kraemer, 2004). Recently, some studies have focused on the effects of RT frequency on muscular adaptations (Arazi \& Asadi, 2011; Dankel et al., 2017; Saric et al., 2018; Gentil et al., 2015). The frequency of RT describe the number of training sessions performed per muscle group in a given period (ACSM, 2009), which is commonly restricted to a week (Dankel et al., 2017).

Previous studies have typically compared 1 vs. 2, 1 vs. 3, 3 vs. 4, and 3 vs. 6 times per week RT frequencies on muscular adaptations, with controversial findings (Arazi \& Asadi, 2011; Dankel et al., 2017; Saric et al., 2018; Gentil et al., 2015, 2018; Brigatto et al., 2018; Colquhoun et al., 2018; Gomes et al., 2018; Hakkinen et al., 1994; Raastad et al., 2012; Zaroni et al., 2019;

67 Schoenfeld et al., 2015; Yue et al., 2018). For example, when Colquhoun et al. (2018) and Saric et al. (2018) compared 3 vs. 6 days per week RT on muscular adaptations in resistance-trained men, with volume equated, both frequencies induced similar gains in strength and muscle hypertrophy. In addition, Brigatto et al. (2018) concluded that both one and two RT session per week promoted neuromuscular adaptations including muscular strength and endurance with a similar change between experimental conditions. Similarly, other authors reported similar changes in muscle strength and hypertrophy with equal volume RT performed one or two times per week in untrained (Gentil et al., 2015) and trained men (Gentile et al., 2018). In contrast, 
Zaroni et al. (2019) examined well-trained men, with a split training routine with muscle groups trained once per week vs. whole-body split training routine with muscle groups trained five days per week, and found that higher frequencies induced superior hypertrophic effect. Moreover, in a series of systematic review studies by Schoenfeld et al. $(2016,2018)$ the authors addressed that twice weekly RT in more effective than once a week RT to increase muscle hypertrophy.

The controversy between studies may derive from previous limitations among published studies. For example, when RT programs of different frequency are performed under volume-equated conditions, muscle strength gain is similar between different frequencies (ACSM, 2009; Schoenfeld et al., 2016, 2018). Another caveat in the literature is that comparisons are usually limited to muscle strength and hypertrophy (Saric et al., 2018; Brigatto et al., 2018; Gomes et al., 2018; Zaroni et al., 2019; Schoenfeld et al., 2015), and little is known about the effects of RT frequencies on muscle power and endurance performance in recreationally trained individuals. Moreover, randomized-controlled interventions, with an equated volume load between different training frequencies are lacking. Therefore, the purpose of this study was to investigate the effects of volume load-equated RT frequencies of 2 vs. 4 times per week on muscular strength, endurance, power performances, and muscle size in recreationally trained young men.

\section{METHODS}

\section{Study design}

94 In a randomized-controlled longitudinal design, subjects were divided into 3 groups, including 5 RT performed 2 times per week (RT2), 4 times per week (RT4) and a control group (CG). The study duration lasted 12 weeks (Figure 1). The main training intervention period lasted 8 weeks and the subjects performed equal volume training with differing training frequencies (i.e., 2 vs. 4 
98 times per week). Pre, mid and post 8-week training, one repetition maximum (1RM) of leg press, 99 bench press, and arm curl, muscular endurance (i.e., 60\% of 1RM to failure) for the upper- and 100 lower-body (i.e., bench press and leg press), countermovement jump and medicine ball throw, in 101 addition to thigh, chest and arm circumferences were measured. Two measurements with $96 \mathrm{~h}$ 102 apart were used to determine the reliability of tests and the intraclass correlation coefficient 103 (ICC) of all tests were $r \geq 0.95$.

$$
* * * \text { Figure } 1 \text { around here } * * *
$$

\section{Participants}

Thirty-five young men who recreationally trained RT (i.e., 2 or 3 days per week for at least 2 years) participated in this study. Inclusion criterions for the study were (1) no upper- and lowerbody injuries or orthopedic problems as screened by physician, (2) no medical problems or any history of ankle, knee, or back pathology in the 3 months before the study, (3) no lower or upperbody reconstructive surgery of any type in the past 2 years or unresolved musculoskeletal disorders, (4) no problems of the cardiovascular and endocrine systems. Furthermore, the subjects were required to not have used any supplement or drug within the past 6 months prior to inclusion in this study which was confirmed by a personal interview. The subjects were assigned to 3 groups including: 2 times per week RT $(\mathrm{RT} 2 ; \mathrm{n}=12$, age $=19.8 \pm 1.8 \mathrm{y}$, height $=1.75 \pm 0.5$ $\mathrm{m}$, mass $=64.2 \pm 5.7 \mathrm{~kg}$, body fat $=16.6 \pm 4.9 \%$, and training age $=2.5 \pm 0.5 \mathrm{y}), 4$ times per week RT $(\mathrm{RT} 4 ; \mathrm{n}=13$, age $=19.9 \pm 1.6 \mathrm{y}$, height $=1.77 \pm 0.4 \mathrm{~m}$, mass $=70.6 \pm 8.2 \mathrm{~kg}$, body fat

$117=18.0 \pm 4.1 \%$, and training age $=2.8 \pm 0.7 \mathrm{y})$ and a control group $(\mathrm{CG} ; \mathrm{n}=10$, age $=20.4 \pm 1.4$ $118 \mathrm{y}$, height $=1.78 \pm 0.8 \mathrm{~m}$, mass $=69.1 \pm 8.0 \mathrm{~kg}$, body fat $=18.4 \pm 3.7 \%$, and training age $=2.3 \pm$ $1190.4 \mathrm{y})$ using computer-generated random numbers (Figure 2). After being informed about the 120 study procedures, benefits and possible risks, the participants signed an informed consent form in 
121 accordance with the guidelines of the Institutional Review Board at the University of Guilan 122 (Project.1398/2019).

\section{Procedures}

126 The volunteers visited the laboratory 9 times for testing including 3 days for pre-test $(24 \mathrm{~h}$ apart 127 between testing sessions), 3 days for mid-test ( $24 \mathrm{~h}$ apart between testing sessions), and 3 days 128 for post-test ( $24 \mathrm{~h}$ apart between testing sessions). The subjects were tested at the same time of 129 day (4 to 6 P.M) and in the same order to minimize the effect of circadian variations in the test results. All subjects were instructed to continue with their normal daily life activities and dietary

131 intake throughout the study duration.

\section{Anthropometric measures}

133 Height was measured using a wall-mounted stadiometer (Seca 222, Terre Haute, IN), body mass 134 was measured using a medical scale (Tanita, BC-418MA, Tokyo, Japan), and skinfold thickness was measured at 3 sites (i.e., pectoral, quadriceps, and abdominal) on the right side of the body using calipers (Lafayette Caliper, model 01128, USA) (Jackson \& Pollock, 1985). Each site measurement was assessed 3 times and the average of 3 trials was recorded for analysis. The circumferences of chest, mid-thigh, and mid-arm on the right side of the body were assessed were measured with the muscle maximally contracted. All anthropometric measures were assessed by the same researcher who was experienced and qualified for the measurements.

\section{Diet control}


143 To avoid potential dietary confounding of results, 3-day diet recalls were completed at pre- and

144 mid study duration, and the subjects were advised to maintain their customary nutritional

145 regimen (i.e., approximately $25 \%$ protein, $25 \%$ fat and 50\% carbohydrate) and to avoid taking

146 any supplements during the study period. The nutrition specialist continued to meet with the

147 subjects each week to assess adherence to their food and liquid instructions and avoidance of

148 drugs and ergogenic supplements using interview before the initiation of each training session 149 (Table 1).

150 Muscular strength

151 Lower body muscular strength was assessed with the leg press exercise, upper-body muscular

152 strength was assessed using the free-weight barbell bench press and arm curl exercises,

153 respectively. The one repetition maximum (1RM) testing was performed according to method 154 previously described in detail (Arazi et al., 2013; Fleck \& Kraemer, 2004). Briefly, the subjects 155 performed a warm-up set of 8 to 10 repetitions at a light weight ( $50 \%$ of $1 \mathrm{RM})$. A second 156 warm-up consisted a set of three to five repetitions with a moderate weight $(\sim 75 \%$ of $1 \mathrm{RM})$, and 157 third warm-up included one to three repetitions with a heavy weight ( $90 \%$ of $1 \mathrm{RM})$. After the 158 warm-up, each subject was tested for the 1RM by increasing the load during consecutive trials 159 until the subjects were unable to perform a proper lift, complete the range of motion, and/or 160 maintain correct technique. The $1 \mathrm{RM}$ test was determined by $\sim 5$ sets of one repetition, with $3-5$ 161 minutes of rest among attempts.

162 A bilateral leg press test was selected to provide data on maximal dynamic strength through the 163 full range of motion of the muscles involved. Bilateral leg press tests were completed using 164 standard a $45^{\circ}$ leg press machine (Nebula Fitness, Inc., Versailles, OH), with the subjects 165 assuming a sitting position (about $120^{\circ}$ flexion at the hips, $80^{\circ}$ flexion at the knees, and $10^{\circ}$ 
166 dorsiflexion) and the weight sliding obliquely at $45^{\circ}$. A manual goniometer (Q-TEC Electronic

167 Co. Ltd., Gyeonggi-do, S. Korea) was used at the knee to standardize the range of motion. On

168 command, the subjects performed a concentric leg extension (as fast as possible) starting from

169 the flexed position $\left(85^{\circ}\right)$ to reach the full extension of $180^{\circ}$ against the resistance determined by

170 the weight. The free-weight barbell (DHZ Barbell Model, Tehran, Iran) bench press is a valid

171 and specific method to assess upper-body strength performance. This test initiated with the arms

172 fully extended, holding the weight directly above the chest. The weight is lowered at a controlled

173 speed and with a smooth motion, to just touch the chest then returned to the starting position.

174 The free-weight barbell (DHZ Barbell Model, Tehran, Iran) arm curl is used as a valid method to

175 assess hand muscle strength. This test initiated in standing position holding barbell using two

176 hands with the arms hanging by the side of body. The elbows were in extending position and

177 then the elbows are closed up to shoulder level while contracting the biceps muscle. The spotters

178 and an experienced strength and conditioning coach provided verbal encouragement and ensure 179 safety.

180 Muscular endurance

181 Before the endurance test, the subjects performed a short period of warm-up including 5 min of 182 running and 5 min of stretching exercise and then performed 10 repetitions with $30-40 \%$ of 1 RM 183 for each exercise test. The muscular endurance tests were performed according to method 184 previously described in detail (Arazi et al., 2014). Briefly, after warm-up, the subjects performed 185 as many repetitions as possible without stopping or pausing between repetitions with $60 \%$ of 186 1RM to exhaustion with 1 hour rest between the two tests (i.e., bench press and leg press) (Arazi $187 \&$ Asadi, 2011).

188 Lower and upper body power performance 
189 Lower body power performance was measured at first, using the countermovement jump test

190 (CMJ). For the CMJ, subjects performed standard warm-up including 10 min light running and

191 ballistic movements and then performed five CMJs without arms akimbo with 30-s rest period

192 (Arazi et al., 2014). The Vertec (Ergo Jump Plus Bosco System, Muscle LabV718, Langesund,

193 Norway) was adjusted to match the height of the individual participant by having him stand with

194 the dominant side to the base of the testing device. The dominant hand was raised and the Vertec

195 was adjusted so that the hand was the appropriate distance away from the marker based on

196 markings on the device itself. The subjects were instructed to flex their knees until $90^{\circ}$ according

197 to previously established methods (Arazi et al., 2014). Each subject performed 3 maximal CMJ

198 with 30-s rest period and the greatest jump recorded for further analysis.

199 Upper body power performance was measured 30 min post CMJ test, using the medicine ball 200 throw (MBT). For the MBT, subjects performed standard warm-up including 10 min of light

201 stretching and ballistic movements for the upper body and then performed five balls throwing 202 with 30-s rest period. The subjects sat on the floor and flexed their elbow similar to basketball 203 chest pass and push the ball (3 kg Rubber Medicine Ball, Champion Sports, Taiwan) as far as 204 possible. There was a floor-mounted tape measure that was used to record distance from sitting 205 position to first contact of the ball. In fact, the distance of the throw of the medicine ball from sat 206 position up to its first contact with the ground was measured as upper body power. Each subject 207 performed five maximal MBT with 30-s rest period (Abe et al., 2000) and the greatest distance 208 recorded for further analysis.

\section{Resistance training program}

210 Table 2 presented the summary of the RT program. The training protocol included a mixture of 211 single-joint and multi-joint exercises with equated training volume load (repetitions $\times$ external 
212 load $[\mathrm{kg}])$ between experimental groups. A 60 to $90 \mathrm{sec}$ period of rest between sets and 2 to 3

213 min of rest between exercises were allowed. The RT intensity was between 70 to $80 \%$ of $1 \mathrm{RM}$

214 which determined by $1 \mathrm{RM}$ testing prior to inclusion in study schedule and weight was increased

215 systematically if the prescribed amount of repetitions were completed. Each training session was

216 supervised by a researcher and Certificated Strength and Conditioning Specialist, with a coach:

217 trainee ratio of 1:5 (Gentil \& Bottaro, 2013). To continuously provide appropriate loading based

218 on the current strength levels of the subjects, they tested at pre-training and after 4 weeks of 219 training to modify RT intensity.

*** Table 2 around here $* * *$

221

222 Statistical analyses

223 A two-way analysis of variance with repeated measures (3 [group] x 3 [time]) was used to

224 determine significant differences among groups. Assumptions of sphericity were assessed using 225 Mauchly's test of sphericity, with any violations adjusted by use of the Greenhouse-Geisser 226 (GG) correction. When a significant F value was achieved, Bonferroni post hoc procedures were 227 performed to identify the pairwise differences between the means. Customized excel spread 228 sheets were used to calculate all effect size (ES) statistics. Hedge's g (g = (Mpost - Mpre) / 229 SDpooled) was utilized to calculate an effect size for all measures. Threshold values for 230 assessing magnitudes of ES were $<0.2$, trivial; 0.2-0.6, small; 0.6-1.2, moderate; 1.2-2.0, large; 231 2.0-4.0, very large; and >4.0, nearly perfect (Hopkins et al., 2009). The effect size is reported 232 with the $95 \%$ confidence interval (CI) for all analysed measures. All data are presented as mean $233 \pm \mathrm{SD}$. The ICC was used to determine the reliability of the measurements. The level of 
234 significance was set at $P \leq 0.05$. The statistical tests were performed using the SPSS statistical

235 package version 21 (Chicago, IL, USA).

\section{RESULTS}

237 The test-retest reliability coefficient of all variable tests was $r \geq 0.95$. At baseline, no significant

238 differences were observed among groups in any dependent variables $(P=0.642)$. In addition, the

239 CG did not show significant changes at any time point in the variables $(P=0.211)$.

240 There was no significant difference between the RT2 (week $4=45.37 \pm 5.62 \mathrm{~kg}$, week $8=91.37$

$241 \pm 11.51 \mathrm{Kg}$ ) and RT4 (week $4=48.68 \pm 6.77 \mathrm{~kg}$, week $8=93.28 \pm 12.42 \mathrm{Kg}$ ) in the training

242 volume load at week $4(P=0.52)$ and week $8(P=0.46)$.

243 There were significant time effects which indicated significant increases in chest and thigh

244 circumferences at mid and post-training intervention for both the RT2 and RT4 $(P=0.01)$. No

245 significant increase was seen in the arm circumference for both the groups $(P=0.6)$. There were

246 significant group by time interaction in chest $(P=0.018)$ and thigh $(P=0.026)$ circumference

247 increases following 8 weeks of training which indicated significant differences between trained

248 groups than CG at mid and post-test values. However, no significant differences were observed

249 between RT2 and RT4 in chest and thigh circumferences at mid and post-test (Table 3 and 4).

250

***Table 3 and 4 around here***

251 There were time effects which indicated significant increases in 1RM of bench press, leg press

252 and arm curl at mid and post-training intervention for both the RT2 and RT4 $(P=0.001)$. There

253 were group by time interaction in $1 \mathrm{RM}$ of bench press $(P=0.031)$ and arm curl $(P=0.022)$

254 following 8 weeks of training which indicated statistically significant differences between the

255 RT4 compared with RT2 at post-test. Compared with CG, both the RT2 and RT4 groups 
256 indicated significant differences at mid- and post-test $(P=0.001)$ in all strength measures (Table $257 \quad 3$ and 4$)$.

258 There was a time effect which indicated significant increases in leg press endurance at mid and 259 post-training intervention for both the RT2 and RT4 $(P=0.001)$. There was a significant group 260 by time interaction $(P=0.041)$ in leg press endurance which indicated significant increases

261 between the trained groups than the CG at mid and post-test values. However, no significant 262 differences were observed between RT2 and RT4 in leg press endurance at mid and post-test 263 (Table 2 and 3). In bench press endurance, there was a time effect which indicated significant 264 increases at mid and post-training intervention for the RT4 $(P=0.001)$. There was a significant 265 group by time interaction $(P=0.032)$ in bench press endurance which indicated significant 266 differences between the RT4 than the CG at mid and post-test values (Table 2 and 3). However, 267 no significant differences were observed between RT2 and RT4 in bench press endurance at mid 268 and post-test (Table 2 and 3).

269 There were time effects which indicated significant increases in CMJ and MBT at mid and post270 training intervention for both the RT2 and RT4 $(P=0.02)$. There were significant group by time 271 interaction $(P=0.04)$ in $\mathrm{CMJ}$ and MBT which indicated significant differences between the

272 trained groups than the CG at mid and post-test values (Table 2 and 3). However, no significant 273 differences were observed between the RT2 and RT4 in CMJ and MBT at mid and post-test 274 (Table 3 and 4).

\section{DISCUSSION}

276 The aim of the present study was to examine the effects of an 8-week RT program performed

277 two or four times per week RT with equal weekly training volume on thigh, arm, and chest 278 circumferences, 1RM of back squat, bench press, and arm curl, muscular endurance and 
279 explosive actions performance for the upper- and lower-body in recreationally trained young 280 men.

281 In circumference measures, both the training groups significantly increased from pre-to-post RT 282 intervention in the chest and thigh circumferences, without significant change for the arm

283

284

285

286

287

288

290

291

292

293

294

295

296

297

298

299

300

301

circumference. In addition, the gains in this marker of muscle size were similar between the RT2 and RT4 groups (small to moderate ES, Table 3), with the exception of pre-to-mid and pre- topost, where the RT2 group that indicated moderate ES while the RT4 group indicated small ES without statistically significant differences. The findings of the present study are in accordance with other studies that have reported improvements in muscle size after RT with varied training frequencies (Arazi \& Asadi, 2011; Saric et al., 2018; Colquhoun et al., 2018; Schoenfeld et al., 2016, 2018). In relation to the effects of training frequency on changes in muscle size or muscular hypertrophy, Schoenfeld et al. $(2016,2018)$ and Grgic et al. (2018) reported small (i.e., range between $\mathrm{ES}=0.22$ to 0.51 ) gains using different $\mathrm{RT}$ frequencies, while in this study we found moderate ( 0.75 to $0.77 \mathrm{ES}$ ) gains in chest circumference after both RT2 and RT4. Previous experimental studies reported that RT interventions with two sessions per week induced small gains (i.e., $0.33 \mathrm{ES}$ ) but in this study we found moderate (range between 0.62 to $0.77 \mathrm{ES}$ ) increases in arm, thigh and chest muscle size. This suggest that RT with a frequency of at least 2 days per week is adequate to enhance muscle size (Gentil et al., 2015, Colquhoun et al., 2018; Zaroni et al., 2019; Yue et al., 2018). The RT2 group performed 4 sets per exercise in each training session, which may induce stimulation of muscular hypertrophy, by signalling pathways that increase protein synthesis and providing mechanical stress in the muscle fibers (Fernandes et al., 2012 Padilha et al., 2019). However, it seems that the muscle hypertrophy expansion is more impressed by volume of training and, considering that both groups trained at what has been 
302 shown to be the optimal dose (Barbalho et al., 2019), it can be derived that frequency of RT

303 might play a subsidiary figure relevant to this and further investigations are needed to illuminate

304 the effects of training frequency under volume-equated conditions on muscle size. In addition,

305 whilst circumference measures has been shown to be reliable and reproducible and might be an

306 appropriate field-centred criterion (de Franca et al., 2015), to make careful deductions based on

307 the evidence, subsequent studies should focus on the use of direct gauges of muscle mass

308 increase using MRI, DXA, ultrasound or BIA; however, previous studies used the

309 aforementioned equipment and reported small gains in muscle hypertrophy using different

310 training frequencies (Gentil et al., 2015, Colquhoun et al., 2018; Zaroni et al., 2019; Yue et al.,

311 2018; Schoenfeld et al., 2016, 2018).

312 Both RT groups increased their 1RM after 4 and 8 weeks training intervention. To date, a large

313 number of studies reported that RT is an optimum training modality for strength enhancement in

314 men and women (Abe et al., 2000; Arazi et al., 2013, 2014). In relation to strength gains

315 following the first 4 weeks of training, aside from muscular hypertrophy, neuromuscular changes

316 may have taken place (i.e., inter-muscular consonance ameliorations, augmented alpha motor-

317 neurons firing rate, modified mechanical specifications of the muscle-tendon complex,

318 ordonnance and/or individual-fiber mechanics) (Loenneke et al., 2019).

319 The RT4 group gained significantly greater strength than the RT2 in 1RM of bench press and 320 arm curl following 8 weeks of training. However, with comparing ES the RT2 indicated large 321 and very large changes in 1RM of leg press following 4 and 8 weeks training intervention. Grgic 322 et al. (2018) in the review article addressed that muscular strength is increased due to more 323 training frequencies; however, RT frequency does not show meaningful effect on muscular 324 strength improvements while equated training volume. They reported moderate ES for 2 and 4 
325 times per week RT frequency (i.e., 0.83 and 1.08, respectively), whereas we found similar gains

326 in bench press and arm curl but large and very large ES in the 1RM leg press. The possible

327 discrepancy in results could be due to type of test measures such as multi-joint vs. single joint

328 (i.e., leg press vs. knee extension) and upper vs. lower body tests. Another possible mechanism

329 for the greater strength gains in bench press and arm curl 1RM after the RT4 compared to the

330 RT2 could be due to motor learning viewpoint. In fact, multi-joint motions including more mixed

331 RT exercises need to an accurate coordination and timing of muscle recruitment and a greater

332 grade of motor efficiency (Carroll et al., 2001). Therefore, increases in RT frequency from 3 to 4

333 sessions per week would provide more exposure to a given test/exercise, which can lead to a

334 higher performance on that test (Mattocks et al., 2017) and hence resulted in greater upper body

335 strength gains in the RT4 group.

336 Our findings demonstrated significant changes in lower and upper-extremity muscular endurance

337 for the RT2 and RT4 groups after 4 and 8 weeks training intervention. These results are

338 according to the last studies that displayed improvements in muscular endurance following RT

339 (Aagaard et al., 2002; Arazi et al., 2013). When comparing the ES, the RT4 showed more gains

340 than RT2 in the endurance tests belonging to leg press and bench press (Table 3). The possible

341 explanation for these findings could be due to the greater possibility of higher frequencies to

342 enhance cellular adaptations (i.e., mitochondrial biogenesis) to increase muscle endurance;

343 however, the information respective to this issue is rare and further studies are needed to explain

344 the influence of different RT frequencies on muscular endurance performance.

345 Both RT groups increased their upper- and-lower body power performance after 4 and 8 weeks

346 training intervention. In line with the results of this study, previous studies reported

347 improvements in power performance after RT (Arazi \& Asadi, 2011; Arazi et al., 2014). 
348 Typically, increases in CMJ and MBT performance following first 4 weeks of training and

349 continually to 8 weeks training intervention could induced by neuromuscular adaptations

350 (Aagaard et al., 2000). In fact, an improvement in power performance in the early stages of a

351 strength training program is likely the result of adaptations in the nervous system (Assuncao et

352 al., 2016). In fact, Aagaard et al. (2000) reported that the principal components of the training

353 enforced progressions following RT were elucidated by elevations in efferent neural drive. This

354 may be one explanation for the changes in lower- and upper-body power performance (i.e., CMJ

355 and MBT) after the RT intervention. However, this is the first study that compared the effects of

356 an 8 week RT with either 2 or 4 weekly training sessions on upper and lower-body power

357 performance. The distribution of training volume on either 2 or 4 weekly training sessions

358 yielded similar effects on power performance for stretch-shortening cycle tasks in CMJ and

359 MBT tests. The observed performance enhancements could be explained by inter-muscular

360 coordination improvements, increased alpha motor-neurons firing rate, improved mechanical

361 characteristics of the muscle-tendon complex, improved muscle size, architecture and/or single-

362 fiber mechanics (Arazi \& Asadi, 2011; Arazi et al., 2014); however, more studies are needed to

363 clarify the impact of training frequencies on power related performance adaptation following RT.

\section{Conclusion}

365 RT improved muscle strength, power performance and markers of muscle size in recreationally

366 trained men; however, four sessions of resistance training per week produced greater gains in

367 muscular strength when compared to two session per week under volume load-equated 368 conditions. Two and four times per week RT induced significant effects on muscular adaptations

369 following 8 weeks of training in recreationally trained young men. In addition, RT for 4 times

370 per week induced further adaptive responses in muscular strength in bench press and arm curl. It 
371 can be recommended that strength and conditioning professionals keep in their mind that 4 times

372 a week RT could be adequate for muscular strength gains and 2 times a week RT could be

373 suitable for the muscle size and power performance under volume load-equated conditions.

374 Availability of data and materials

375 The data of the current study are available as supplemental file.

376 Competing interests

377 The authors declare that they have no competing interests.

\section{$378 \quad$ Funding}

379 This research has not received any financial support.

380

381

382

383

384

\section{Authors' contributions}

HA and AA designed the study. PJ and AG performed the experiment. HA, AA, PJ, AG, RRC, and PG analysed the data and wrote the manuscript. HA, AA, RRC, PG, ACH and HZ involved in the interpretation of data, reviewed and edited the manuscript. All authors read and approved the final manuscript.

\section{Acknowledgments}

The authors are thankful to the participants of this study for their excellent collaborations.

\section{References}

Aagaard P, Erik B, Jesper S, Andersen L, Magnusson P, Dyhre-Poulsen P. 2002. Increased rate of force development and neural drive of human skeletal muscle following resistance training. Journal of Applied Physiology 93:1318-1326.

Abe T, DeHoyos DV, Pollock ML, Garzarella L. 2000. Time course for strength and muscle thickness changes following upper and lower body resistance training in men and women. European Journal of Applied Physiology and Occupational Physiology 81:174-180 ACSM. 2009. American College of Sports Medicine position stand. Progression models in resistance training for healthy adults. Medicine and Science in Sports and Exercise 41:687-708 
399

400

401

402

403

404

405

406

407

408

409

410

411

412

413

414

415

416

417

418

419

420

421

422

423

424

425

426

427

428

429

430

431

432

433

434

435

436

437

438

439

440

441

442

443
Arazi H, Asadi A. 2011. Effects of 8 weeks equal-volume resistance training with different workout frequency on maximal strength, endurance and body composition. International Journal of Sports Science and Engineering 5:112-118.

Arazi H, Asadi A, Roohi S. 2014. Enhancing muscular performance in women: compound versus complex, traditional resistance and plyometric training alone. Journal of Musculoskeletal Research 17:1450007, 1-10.

Arazi H, Damirchi A, Asadi A. 2013. Age-related muscle circumference, strength development and hormonal adaptations with 8 weeks moderate intensity resistance training. Annals de Endocrinology 74:30-35.

Assunção AR, Bottaro M, Ferreira-Junior JB, Izquierdo M, Cadore EL, Gentil P. 2016. The Chronic effects of low- and high-intensity resistance training on muscular fitness in adolescents. PLoS One 11(8).

Barbalho M, Coswig VS, Steele J, Fisher JP, Giessing J, Gentil P. 2019. Evidence of a ceiling effect for training volume in muscle hypertrophy and strength in trained men - less is more? International Journal of Sports Physiology and Performance 12:1-23 Epub ahead of print.

Brigatto FA, Braz TV, Zanini T, Germano MD, Aoki MS, Schoenfeld BJ. 2018. Effect of resistance training frequency on neuromuscular performance and muscle morphology after eight weeks in trained men. Journal of Strength and Conditioning Research doi: 10.1519/ JSC.0000000000002563. Epub ahead of print.

Carroll TJ, Riek S, Carson RG. 2001. Neural adaptations to resistance training: implications for movement control. Sports Medicine 31:829-840.

Colquhoun RJ, Gai CM, Aguilar D, Bove D, Dolan J, Vargas A. 2018. Training volume, not frequency, indicative of maximal strength adaptations to resistance training. Journal of Strength and Conditioning Research 32:1207-1213.

Dankel SJ, Mattocks KT, Jessee MB, Buckner SL, Mouser JG, Counts BR, Laurentino GC, Loenneke JP. 2017. Frequency: the overlooked resistance training variable for inducing muscle hypertrophy? Sports Medicine 47:79-805

de França HS, Branco PA, Guedes Junior DP, Gentil P, Steele J, Teixeira CV. 2015. The effects of adding single-joint exercises to a multi-joint exercise resistance training program on upper body muscle strength and size in trained men. Applied Physiology Nutrition and Metabolism 40:822-826.

Fernandes T, Úrsula PR, Soci Stéphano FS, MeloCléber R, Alves Edilamar M. 2012. Signaling pathways that mediate skeletal muscle hypertrophy: effects of exercise training, skeletal muscle. Myogenesis to Clinical Relations, Julianna Cseri, IntechOpen. 
444

445

446

447

448

449

450

451

452

453

454

455

456

457

458

459

460

461

462

463

464

465

466

467

468

469

470

471

472

473

474

475

476

477

478

479

480

481

482

483

484

485

486

487

488

489

490

Fleck SJ, Kraemer WJ. 2004. Designing Resistance Training Programs, 3nd Ed. Champaign, IL: Human Kinetics.

Gentil P, Bottaro M. 2013. Effects of training attendance on muscle strength of young men after 11 weeks of resistance training. Asian Journal of Sports Medicine 4:101-106.

Gentil P, Fischer B, Martorelli AS, Lima RM, Bottaro M. 2015. Effects of equal-volume resistance training performed one or two times a week in upper body muscle size and strength of untrained young men. Journal of Sports Medicine and Physical Fitness 55:144-149.

Gentil P, Fisher J, Steele J, Campos MH, Silva MH, Paoli A. 2018. Effects of equal-volume resistance training with different training frequencies in muscle size and strength in trained men. Peer J 6:e5020.

Gomes GK, Franco CM, Nunes PRP, Orsatti FL. 2018. High frequency resistance training is not more effective than low frequency resistance training in increasing muscle mass and strength in well-trained men. Journal of Strength and Conditioning Research doi: 10.1519/ JSC.0000000000002559.

Grgic J, Schoenfeld BJ, Latella C. 2018. Resistance training frequency and skeletal muscle hypertrophy: A review of available evidence. Journal of Science and Medicine in Sport doi.org/10.1016/j.jsams.2018.09.223.

Grgic J, Schoenfeld BJ, Davies TB, Lazinica B, Krieger JW, Pedisic, Z. 2018. Effect of resistance training frequency on gains in muscular strength: A systematic review and metaanalysis. Sports Medicine 48:1207-1220.

Ha"kkinen K, Kallinen M. 1994. Distribution of strength training volume into one or two daily sessions and neuromuscular adaptations in female athletes. Electromyography and Clinical Neurophysiology 34:117-124.

Hopkins, WG, Marshall, S, and Batterham, A. 2009. Progressive statistics for studies in sports medicine and exercise science. Medicine and Science in Sports and Exercise 41:3-13.

Jackson AS, Pollock ML. 1985. Practical assessment of body composition. Physician and Sports Medicine 13:82-90.

Loenneke JP, Buckner SL, Dankel SJ, Abe T. 2019. Exercise-induced changes in muscle size do not contribute to exercise-induced changes in muscle strength. Sports Medicine 49:987-991.

Mattocks KT, Buckner SL, Jessee MB, Dankel SJ, Mouser JG, Loenneke JP. 2017. Practicing the test produces strength equivalent to higher volume training. Medicine and Science in Sports and Exercise 49:1945-1954.

Padilha CS, Cella PS, Ribeiro AS, Voltaelli FA, Testa MTJ, Marinello PC, Iarosz KC, Guirro PB, Meminice R. 2019. Moderate vs high-load resistance training on muscular adaptations in rats. Life Science 238: 116964. 
491

492

493

494

495

496

497

498

499

500

501

502

503

504

505

506

507

508

509

510

511

512

513

514

515

516

517

518

519

520

521

522

523

524

525

526

527

528

Raastad, T, Kirketeig, A, Wolf, D, and Paulsen, G. 2012. Powerlifters improved strength and muscular adaptations to a greater extent when equal total training volume was divided into 6 compared to 3 training sessions per week. 17th Annual Conference of the ECSS, Brugge, Belgium, July 4-7.

Saric J, Lisica D, Orlic I, Grgic J, Krieger JW, Vuk S, Schoenfeld BJ. 2018. Resistance training frequencies of 3 and 6 times per week produce similar muscular adaptations in resistance-trained men. Journal of Strength and Conditioning Research .doi: 10.1519/JSC.0000000000002909.

Schoenfeld BJ, Grgic J, Krieger J. 2018. How many times per week should a muscle be trained to maximize muscle hypertrophy? A systematic review and meta-analysis of studies examining the effects of resistance training frequency. Journal of Sports Sciences doi:10.1080/02640414.2018.1555906.

Schoenfeld, BJ, Ogborn, D, Krieger, JW. 2016. Effects of resistance training frequency on measures of muscle hypertrophy: A systematic review and meta-analysis. Sports Medicine 46: 1689-1697.

Schoenfeld, BJ, Ratamess, NA, Peterson, MD, Contreras, B, and Tiryaki-Sonmez, G. 2015. Influence of resistance training frequency on muscular adaptations in well-trained men. Journal of Strength and Conditioning Research 29:1821-1829.

Yue FL, Karsten B, Larumbe-Zabala E, Seijo M, Naclerio F. 2018. Comparison of 2 weeklyequalized volume resistance-training routines using different frequencies on body composition and performance in trained males. Applied Physiology Nutrition and Metabolism 43:475-481.

Zaroni R, Brigatto F, Schoenfeld BJ, Braz TV, Camargo J, Germano M. 2019. High resistance training frequency enhances muscle thickness in resistance trained men. Journal of Strength and Conditioning Research 33:140-151.

\section{Figure Legends:}

Figure 1. Study design.

Figure 2. Study flow.

\section{Table Legends:}

Table 1. Dietary intake assessed for the RT2, RT4 and control groups at pre-and in the middle of the training period (mean $\pm \mathrm{SD})$. 
530 Table 2. Resistance training protocol.

531 RT2: 2 times per week resistance training, RT4: 4 times per week resistance training, RM: repetition 532 maximum.

533

534 Table 3. Changes in anthropometric and performance variables in response to 8 weeks training 535 intervention (mean $\pm \mathrm{SD})$.

536 RT2: 2 times per week resistance training, RT4: 4 times per week resistance training, CG: control group.

537 *significant differences compared to pre-value, $\dagger$ significant differences compared to mid-value, $\$$ significant 538 differences compared to $\mathrm{CG},{ }^{* *}$ significant differences between training groups. G: group, T: time.

540 Table 4. Time point ES in anthropometric and performance variables in response to 8 weeks 541 training intervention.

542 RT2: 2 times per week resistance training, RT4: 4 times per week resistance training, CG: control group. a, trivial; b, 543 small; c, moderate; d, large ES. 


\section{Table $\mathbf{1}$ (on next page)}

Dietary intake assessed for the RT2, RT4 and control groups at pre and in the middle of the training period (mean $\pm \mathrm{SD}$ ). 
Table 1. Dietary intake assessed for the RT2, RT4 and control groups at pre and in the middle of the training period (mean \pm SD).

\begin{tabular}{lllll}
\hline & & RT2 & RT4 & Control \\
\hline Energy intake (kcal) & Pre & $2632 \pm 310$ & $2521 \pm 276$ & $2618 \pm 288$ \\
& Mid & $2991 \pm 298$ & $2892 \pm 199$ & $2632 \pm 299$ \\
Carbohydrate (g) & Pre & $270 \pm 33$ & $269 \pm 39$ & $272 \pm 37$ \\
& Mid & $292 \pm 41$ & $298 \pm 41$ & $288 \pm 33$ \\
Fat (g) & Pre & $85 \pm 21$ & $87 \pm 23$ & $81 \pm 33$ \\
& Mid & $94 \pm 24$ & $92 \pm 21$ & $82 \pm 38$ \\
Protein (g) & Pre & $108 \pm 22$ & $105 \pm 21$ & $100 \pm 19$ \\
& Mid & $129 \pm 26$ & $125 \pm 30$ & $98 \pm 22$ \\
Vitamin E (mg) & Pre & $9.6 \pm 1.0$ & $9.3 \pm 1.2$ & $9.1 \pm 1.1$ \\
& Mid & $11.0 \pm 1.5$ & $10.7 \pm 1.4$ & $9.0 \pm 0.8$ \\
Vitamin C (mg) & Pre & $72 \pm 18$ & $71 \pm 17$ & $70 \pm 15$ \\
& Mid & $79 \pm 21$ & $78 \pm 13$ & $71 \pm 18$ \\
\hline
\end{tabular}




\section{Table 2 (on next page)}

Resistance training protocol.

RT2: 2 times per week resistance training, RT4: 4 times per week resistance training, RM: repetition maximum. 
Table 2. Resistance training protocol.

\begin{tabular}{lllll}
\hline RT2 group & Saturday & Repetitions & Tuesday & Repetitions \\
\hline & Leg press & $10-10-8-8$ & Leg extension & $10-10-8-8$ \\
& Lying leg curl & $10-10-8-8$ & Deadlift & $10-10-8-8$ \\
& Lat pull down & $10-10-8-8$ & Lat rowing & $10-10-8-8$ \\
& Bench press & $10-10-8-8$ & Incline bench press & $10-10-8-8$ \\
& Lateral raises & $10-10-8-8$ & Military press & $10-10-8-8$ \\
& Machine biceps curl & $10-10-8-8$ & Arm curl & $10-10-8-8$ \\
& Machine triceps extension & $10-10-8-8$ & Lying triceps extension & $10-10-8-8$ \\
\hline RT4 group & Saturday and Tuesday & Repetitions & Sunday and Wednesday & Repetitions \\
\hline & Leg press & $10-8$ & Leg extension & $10-8$ \\
& Lying leg curl & $10-8$ & Deadlift & $10-8$ \\
& Lat pull down & $10-8$ & Lat rowing & $10-8$ \\
& Bench press & $10-8$ & Incline bench press & $10-8$ \\
& Lateral raises & $10-8$ & Military press & $10-8$ \\
& Machine biceps curl & $10-8$ & Arm curl & $10-8$ \\
& Machine triceps extension & $10-8$ & Lying triceps extension & $10-8$ \\
\hline
\end{tabular}

RT2: 2 times per week resistance training, RT4: 4 times per week resistance training, RM: repetition maximum. 


\section{Table 3 (on next page)}

Changes in anthropometric and performance variables in response to 8 weeks training intervention (mean $\pm \mathrm{SD}$ ).

RT2: 2 times per week resistance training, RT4: 4 times per week resistance training, CG: control group. *significant differences compared to pre-value, $\uparrow$ significant differences compared to mid-value, $\ddagger$ significant differences compared to CG, ** significant differences between training groups. G: group, T: time. 
Table 3. Changes in anthropometric and performance variables in response to 8 weeks training intervention (mean \pm SD).

\begin{tabular}{|c|c|c|c|c|c|}
\hline \multirow[b]{2}{*}{ Variable } & \multirow[b]{2}{*}{ Group } & \multicolumn{3}{|c|}{ Testing time } & \multirow[t]{2}{*}{ Statistics } \\
\hline & & Pre & Mid & Post & \\
\hline \multirow{3}{*}{$\begin{array}{l}\text { Chest circumference } \\
(\mathrm{cm})\end{array}$} & RT2 & $86.4 \pm 4$ & $88.7 \pm 4.3^{*}$ & $89.8 \pm 4.5^{*}+$ & $\mathrm{G}=0.08$ \\
\hline & RT4 & $86.5 \pm 7.3$ & $91.3 \pm 8.6^{*}+$ & $92.5 \pm 8.1 * t$ & $\mathrm{~T}=0.001$ \\
\hline & $\mathrm{CG}$ & $86.3 \pm 6.8$ & $87.1 \pm 7.5$ & $86.8 \pm 7.2$ & $\mathrm{G} \times \mathrm{T}=0.018$ \\
\hline \multirow{3}{*}{$\begin{array}{l}\text { Thigh circumference } \\
\text { (cm) }\end{array}$} & RT2 & $53.6 \pm 3.3$ & $56.0 \pm 3.7 *+$ & $56.1 \pm 3.4^{*}+$ & $\mathrm{G}=0.54$ \\
\hline & RT4 & $54.7 \pm 7.6$ & $56.2 \pm 6.0 * t$ & $56.3 \pm 6.3 * t$ & $\mathrm{~T}=0.001$ \\
\hline & $\mathrm{CG}$ & $53.5 \pm 4.4$ & $52.1 \pm 3.4$ & $51.8 \pm 4.2$ & $\mathrm{G} \times \mathrm{T}=0.026$ \\
\hline \multirow{3}{*}{$\begin{array}{l}\text { Arm circumference } \\
(\mathrm{cm})\end{array}$} & RT2 & $27.2 \pm 2.5$ & $28.2 \pm 2.9$ & $28.8 \pm 2.5$ & $\mathrm{G}=0.1$ \\
\hline & RT4 & $29.3 \pm 3.4$ & $29.8 \pm 3.4$ & $29.5 \pm 3.1$ & $\mathrm{~T}=0.6$ \\
\hline & $\mathrm{CG}$ & $27.6 \pm 2.1$ & $27.0 \pm 1.2$ & $26.6 \pm 1.2$ & $\mathrm{G} \times \mathrm{T}=0.12$ \\
\hline \multirow{3}{*}{$\begin{array}{l}\text { 1RM bench press } \\
\text { (kg) }\end{array}$} & RT2 & $63.5 \pm 6.4$ & $70.3 \pm 7.3 *+$ & $71.5 \pm 10.5 * t$ & $\mathrm{G}=0.12$ \\
\hline & RT4 & $64.6 \pm 5.3$ & $69.8 \pm 10.6^{*}+$ & $75.5 \pm 12.8 *+t * *$ & $\mathrm{~T}=0.001$ \\
\hline & $\mathrm{CG}$ & $64.4 \pm 7.5$ & $63.5 \pm 7.5$ & $64.8 \pm 6.2$ & $\mathrm{G} \times \mathrm{T}=0.031$ \\
\hline \multirow{3}{*}{$\begin{array}{l}\text { 1RM leg press } \\
\text { (kg) }\end{array}$} & RT2 & $201.2 \pm 36.6$ & $260.6 \pm 48.0 * t$ & $310.3 \pm 52.5 *+t$ & $\mathrm{G}=0.48$ \\
\hline & RT4 & $203.4 \pm 51.7$ & $263.9 \pm 68.5^{*}+$ & $299.8 \pm 64.2 *+t$ & $\mathrm{~T}=0.01$ \\
\hline & $\mathrm{CG}$ & $202.8 \pm 45.2$ & $204.1 \pm 39.8$ & $203.4 \pm 41.2$ & $\mathrm{G} \times \mathrm{T}=0.47$ \\
\hline \multirow{3}{*}{$\begin{array}{l}\text { 1RM arm curl } \\
(\mathrm{kg})\end{array}$} & RT2 & $28.7 \pm 4.0$ & $33.6 \pm 4.0 *+$ & $34.3 \pm 8.2 * t$ & $\mathrm{G}=0.1$ \\
\hline & RT4 & $28.6 \pm 5.3$ & $35.8 \pm 6.2 *+$ & $37.2 \pm 8.7 *+* *$ & $\mathrm{~T}=0.001$ \\
\hline & $\mathrm{CG}$ & $29.1 \pm 3.1$ & $29.1 \pm 2.5$ & $29.9 \pm 3.1$ & $\mathrm{G} \times \mathrm{T}=0.022$ \\
\hline \multirow{3}{*}{$\begin{array}{l}\text { Bench press endurance } \\
\text { (repetitions) }\end{array}$} & RT2 & $21.1 \pm 3.6$ & $21.5 \pm 3.6$ & $21.5 \pm 3.8$ & $\mathrm{G}=0.11$ \\
\hline & RT4 & $21.5 \pm 2.6$ & $23.0 \pm 3.1 *+$ & $23.5 \pm 2.3^{*}+$ & $\mathrm{T}=0.01$ \\
\hline & $\mathrm{CG}$ & $19.4 \pm 4.4$ & $19.8 \pm 4.6$ & $20.1 \pm 3.5$ & $\mathrm{G} \times \mathrm{T}=0.032$ \\
\hline \multirow{3}{*}{$\begin{array}{l}\text { Leg press endurance } \\
\text { (repetitions) }\end{array}$} & RT2 & $20.7 \pm 7.0$ & $24.1 \pm 4.2^{*}+$ & $26.5 \pm 5.4 *+$ & $\mathrm{G}=0.07$ \\
\hline & RT4 & $19.4 \pm 4.8$ & $27.0 \pm 4.9 *+$ & $27.2 \pm 6.4 * t$ & $\mathrm{~T}=0.001$ \\
\hline & $\mathrm{CG}$ & $20.2 \pm 3.3$ & $20.8 \pm 5.5$ & $19.3 \pm 4.2$ & $\mathrm{G} \times \mathrm{T}=0.041$ \\
\hline \multirow{2}{*}{$\begin{array}{l}\text { Countermovement jump } \\
\text { (cm) }\end{array}$} & RT2 & $37.3 \pm 4.4$ & $41.7 \pm 5.2 * t$ & $43.7 \pm 3.3 *+$ & $\mathrm{G}=0.36$ \\
\hline & RT4 & $37.8 \pm 4.6$ & $41.6 \pm 3.4 *+$ & $42.8 \pm 5.5^{*}+$ & $\mathrm{T}=0.021$ \\
\hline
\end{tabular}




$\begin{array}{lllllc} & \text { CG } & 37.4 \pm 3.7 & 37.0 \pm 3.9 & 37.1 \pm 4.6 & \mathrm{G} \times \mathrm{T}=0.047 \\ \begin{array}{llll}\text { Medicine ball throw } \\ \text { (meters) }\end{array} & \text { RT2 } & 3.49 \pm 0.52 & 3.64 \pm 0.45^{*} \ddagger & 3.72 \pm 0.37 * \dagger & \mathrm{G}=0.87 \\ & \text { RT4 } & 3.72 \pm 0.5 & 3.86 \pm 0.58^{*} \ddagger & 3.99 \pm 0.65^{*}+ & \mathrm{T}=0.029 \\ \mathrm{G} \times \mathrm{T}=0.048\end{array}$

RT2: 2 times per week resistance training, RT4: 4 times per week resistance training, CG: control group. * significant differences4 compared to pre-value, $\dagger$ significant differences compared to mid-value, $\$$ significant differences compared to CG, $* *$ significant5 differences between training groups. G: group, T: time. 


\section{Table 4 (on next page)}

Time point ES in anthropometric and performance variables in response to 8 weeks training intervention.

RT2: 2 times per week resistance training, RT4: 4 times per week resistance training, CG: control group. a, trivial; b, small; c, moderate; d, large ES. 
Table 4. Time point ES in anthropometric and performance variables in response to 8 weeks training intervention.

\begin{tabular}{|c|c|c|c|c|}
\hline \multirow[b]{2}{*}{ Variable } & \multirow[b]{2}{*}{ Group } & \multicolumn{3}{|c|}{ ES (95\% Cl) } \\
\hline & & Pre to mid & Mid to post & Pre to post \\
\hline \multirow{3}{*}{$\begin{array}{l}\text { Chest circumference } \\
\text { (cm) }\end{array}$} & RT2 & $0.53(-0.28$ to 1.35$) b$ & $0.24(-0.56$ to 1.04$) b$ & $0.77(-0.06$ to 1.6$) \mathrm{c}$ \\
\hline & RT4 & $0.58(-0.2$ to 1.37$) b$ & $0.14(-0.63$ to 0.91$) a$ & $0.75(-0.04$ to 1.55$) \mathrm{c}$ \\
\hline & $\mathrm{CG}$ & $0.11(-0.77$ to 0.98$)$ & $-0.04(-0.92$ to 0.84$)$ & $0.07(-0.81$ to 0.95$)$ \\
\hline \multirow{3}{*}{$\begin{array}{l}\text { Thigh circumference } \\
\text { (cm) }\end{array}$} & RT2 & $0.66(-0.13$ to 1.45$) \mathrm{c}$ & $0.03(-0.74$ to 0.8$) \mathrm{a}$ & $0.72(-0.07$ to 1.52$) \mathrm{c}$ \\
\hline & RT4 & $0.21(-0.59$ to 1.01$) b$ & $0.02(-0.78$ to 0.82$) \mathrm{a}$ & $0.22(-0.58$ to 1.02$) b$ \\
\hline & $\mathrm{CG}$ & $-0.22(-1.1$ to 0.65$)$ & $-0.08(-0.95$ to 0.8$)$ & $-0.38(-1.26$ to 0.51$)$ \\
\hline \multirow{3}{*}{$\begin{array}{l}\text { Arm circumference } \\
(\mathrm{cm})\end{array}$} & RT2 & $0.36(-0.42$ to 1.13$) b$ & $0.21(-0.56$ to 0.99$) b$ & $0.62(-0.17$ to 1.41$) \mathrm{c}$ \\
\hline & RT4 & $0.14(-0.66$ to 0.94$) a$ & $0.09(-0.71$ to 0.89$) a$ & $0.06(-0.74$ to 0.86$) \mathrm{a}$ \\
\hline & $\mathrm{CG}$ & $-0.34(-1.22$ to 0.55$)$ & $-0.32(-1.2$ to 0.56$)$ & $-0.56(-1.45$ to 0.33$)$ \\
\hline \multirow{3}{*}{$\begin{array}{l}\text { 1RM bench press } \\
(\mathrm{kg})\end{array}$} & RT2 & $0.96(0-.15$ to 1.77$) \mathrm{c}$ & $0.13(-0.64$ to 0.9$) \mathrm{a}$ & $0.89(-0.08$ to 1.7$) \mathrm{c}$ \\
\hline & RT4 & $0.6(-0.22$ to 1.42$) b$ & $0.47(-0.34$ to 1.28$) b$ & $1.07(-0.22$ to 1.93$) \mathrm{c}$ \\
\hline & $\mathrm{CG}$ & $-0.11(-0.99$ to 0.76$)$ & $0.18(-0.7$ to 1.06$)$ & $0.06(-0.82$ to 0.93$)$ \\
\hline \multirow{3}{*}{$\begin{array}{l}\text { 1RM leg press } \\
(\mathrm{kg})\end{array}$} & RT2 & $1.35(0.5$ to 2.2$) \mathrm{d}$ & $0.96(0.15$ to 1.77$) \mathrm{c}$ & $2.33(1.34$ to 3.33$) \mathrm{e}$ \\
\hline & RT4 & $0.96(0.12$ to 1.81$) \mathrm{c}$ & $0.52(0.29$ to 1.34$) b$ & $1.6(0.68$ to 2.52$) \mathrm{d}$ \\
\hline & $\mathrm{CG}$ & $0.03(-0.85$ to 0.91$)$ & $-0.02(-0.89$ to 0.86$)$ & $0.01(-0.86$ to 0.89$)$ \\
\hline \multirow{3}{*}{$\begin{array}{l}\text { 1RM arm curl } \\
(\mathrm{kg})\end{array}$} & RT2 & $1.17(0.22$ to 2.12$) \mathrm{c}$ & $0.11(-0.66$ to 0.87$) \mathrm{a}$ & $0.84(-0.04$ to 1.64$) \mathrm{c}$ \\
\hline & RT4 & $1.21(0.34$ to 2.08$) \mathrm{d}$ & $0.18(-0.62$ to 0.98$) a$ & $1.15(0.29$ to 2.02$) \mathrm{c}$ \\
\hline & $\mathrm{CG}$ & $0.0(-0.88$ to 0.88$)$ & $0.27(-0.61$ to 1.15$)$ & $0.25(-0.63$ to 1.13$)$ \\
\hline \multirow{3}{*}{$\begin{array}{l}\text { Bench press endurance } \\
\text { (repetitions) }\end{array}$} & RT2 & $0.11(-0.66$ to 0.88$) a$ & $0.0(-0.77$ to 0.77$) \mathrm{a}$ & $0.1(-0.66$ to 0.87$) a$ \\
\hline & RT4 & $0.51(-0.31$ to 1.32$) b$ & $0.18(-0.62$ to 0.98$) a$ & $0.79(-0.04$ to 1.62$) \mathrm{c}$ \\
\hline & $\mathrm{CG}$ & $0.09(-0.79$ to 0.96$)$ & $0.07(-0.81$ to 0.95$)$ & $0.17(-0.71$ to 1.05$)$ \\
\hline \multirow{3}{*}{$\begin{array}{l}\text { Leg press endurance } \\
\text { (repetitions) }\end{array}$} & RT2 & $0.57(-0.21$ to 1.35$) b$ & $0.48(-0.3$ to 1.26$) b$ & $0.9(-0.09$ to 1.71$) \mathrm{c}$ \\
\hline & RT4 & $1.51(0.61$ to 2.42$) \mathrm{d}$ & $0.03(-0.77$ to 0.83$) \mathrm{a}$ & $1.33(0.45$ to 2.22$) \mathrm{d}$ \\
\hline & $\mathrm{CG}$ & $0.13(-0.75$ to 1$)$ & $-0.29(-1.17$ to 0.59$)$ & $-0.23(-1.11$ to 0.65$)$ \\
\hline \multirow{3}{*}{$\begin{array}{l}\text { Countermovement jump } \\
\text { (cm) }\end{array}$} & RT2 & $0.88(0.08$ to 1.69$) \mathrm{c}$ & $0.44(-0.33$ to 1.22$) b$ & $1.59(0.71$ to 2.48$) \mathrm{d}$ \\
\hline & RT4 & $0.91(0.07$ to 1.75$) \mathrm{c}$ & $0.25(-0.55$ to 1.06$) b$ & $0.95(0.11$ to 1.8$) \mathrm{c}$ \\
\hline & $\mathrm{CG}$ & $0.1(-0.78$ to 0.98$)$ & $0.02(-0.85$ to 0.9$)$ & $0.07(-0.81$ to 0.95$)$ \\
\hline
\end{tabular}




$\begin{array}{lllll}\begin{array}{l}\text { Medicine ball throw } \\ \text { (meters) }\end{array} & \text { RT2 } & 0.3(-0.47 \text { to } 1.07) \mathrm{b} & 0.19(-0.58 \text { to } 0.96) \mathrm{a} & 0.49(-0.29 \text { to } 1.27) \mathrm{b} \\ & \text { RT4 } & 0.25(-0.55 \text { to } 1.05) \mathrm{b} & 0.2(-0.6 \text { to } 1.01) \mathrm{a} & 0.45(-0.36 \text { to } 1.26) \mathrm{b} \\ & \text { CG } & 0.03(-0.85 \text { to } 0.9) & 0.0(-0.88 \text { to } 0.88) & 0.02(-0.85 \text { to } 0.9)\end{array}$

RT2: 2 times per week resistance training, RT4: 4 times per week resistance training, CG: control group.

a, trivial; b, small; c, moderate; d, large; e, very large ES. 
Figure 1

Study design.

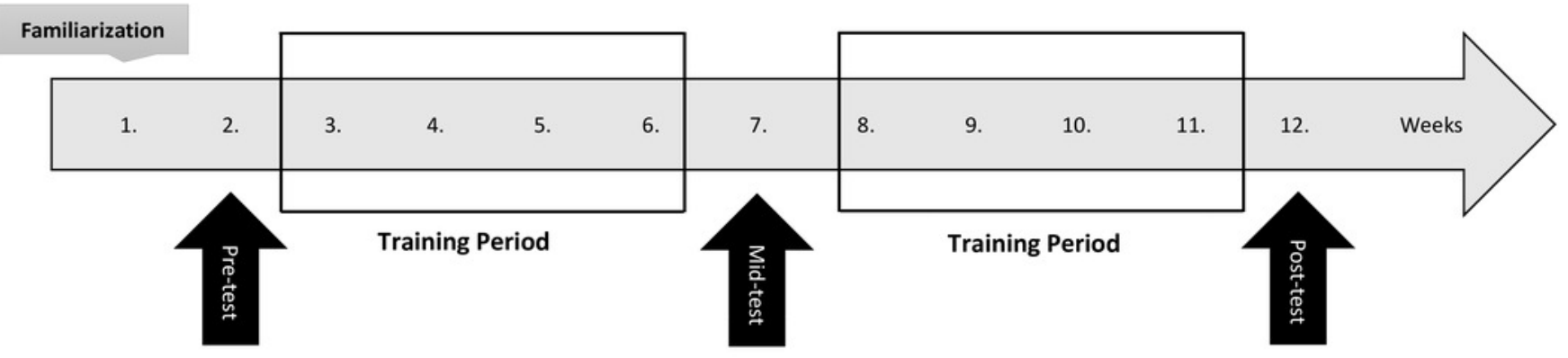


Figure 2

Study flow. 


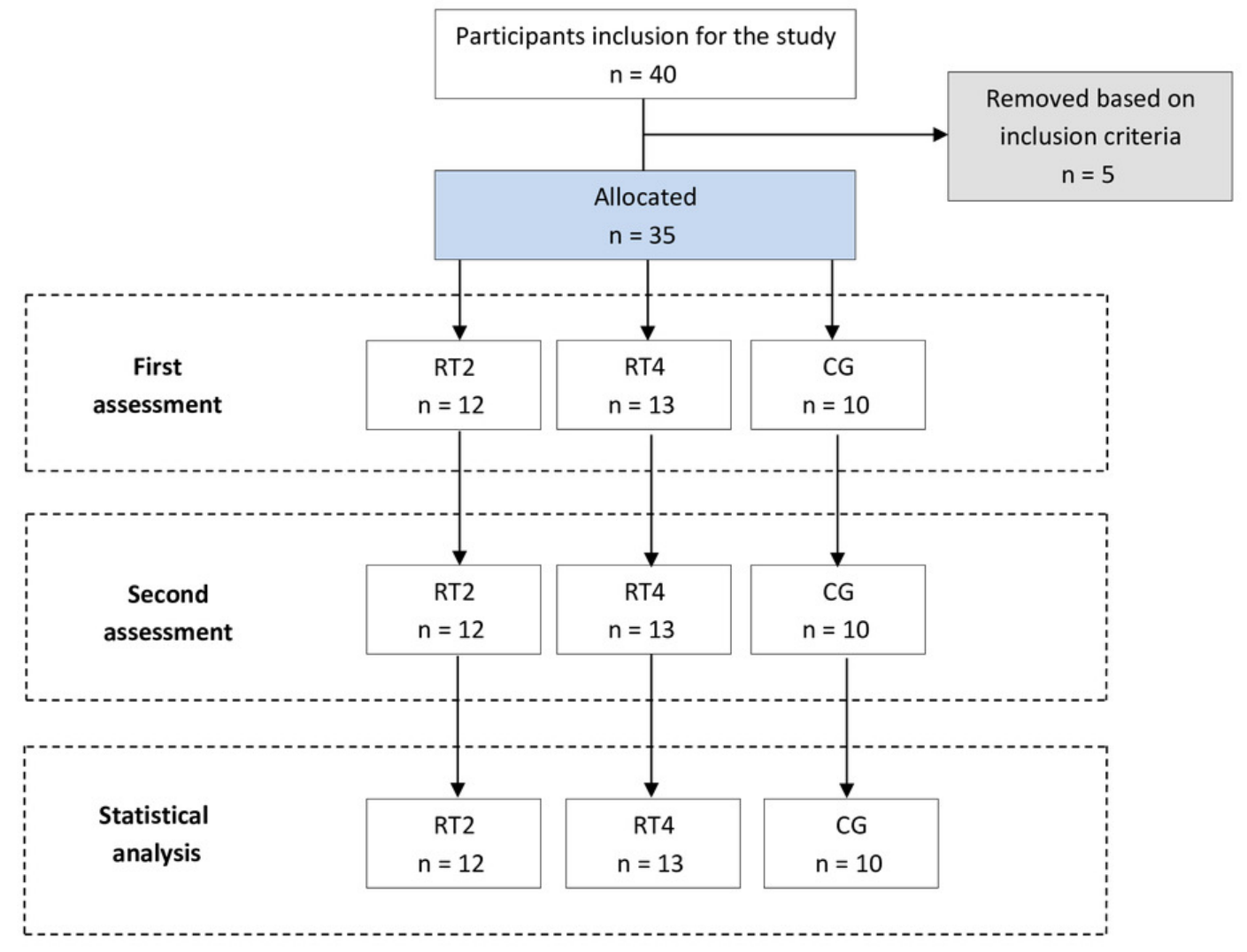

